\title{
Grundvoraussetzungen herzchirurgischer Einheiten zur Behandlung von Patienten mit angeborenen Herzfehlern
}

\section{Konsensuspapier der Vorstände der Deutschen Gesellschaft für Thorax-, Herz- und Gefäßchirurgie (DGTHG) und der Deutschen Gesellschaft für Pädiatrische Kardiologie (DGPK)}

\author{
Deutsche Gesellschaft für Thorax-, Herz- und Gefäßchirurgie (DGTHG), Berlin, Germany \\ Deutsche Gesellschaft für Pädiatrische Kardiologie (DGPK), Düsseldorf, Germany
}

\section{Zusammenfassung}

Diese Publikation definiert grundlegende Strukturen für herzchirurgische Einheiten zur Behandlung angeborener Herzfehler in Deutschland. Sie wurde von den Vorständen der Deutschen Gesellschaft für Thorax-, Herz- und Gefäßchirurgie (DGTHG) und der Deutschen Gesellschaft für Pädiatrische Kardiologie (DGPK), in Zusammenarbeit mit der Arbeitsgemeinschaft Chirurgie angeborener Herzfehler und Kinderherzchirurgie, erarbeitet.

Dieses aktualisierte Konsensuspapier beruht in Grundzügen auf einer Veröffentlichung der European Association for Cardiothoracic Surgery (EACTS) und ist eine Aktualisierung und Weiterentwicklung des 2005 erstmals publizierten Strukturpapiers der DGTHG. Einrichtungen für die kinderkardiologische und herzchirurgische Versorgung von Patienten mit angeborenen Herzfehlern unterliegen in Deutschland diversen Anforderungen. Beispielsweise wurde im Jahr 2010 durch den Gemeinsamen Bundesausschuss (G-BA) die Richtlinie über Maßnahmen zur Qualitätssicherung der herzchirurgischen Versorgung bei Kindern und Jugendlichen (Richtlinie Kinderherzchirurgie) in Kraft gesetzt, die Anforderungen an die Struktur- und Prozessqualität verbindlich regelt. Bis heute bestehen auch weiterhin grundlegende und erhebliche Unterschiede der in diesem Zusammenhang verfügbaren Versorgungseinrichtungen.

Herzchirurgische Einheiten zur Behandlung angeborener Herzfehler bei Kindern und Jugendlichen müssen das gesamte herzchirurgische Behandlungsspektrum vom Frühgeborenen bis zum Jugendlichen mit angeborenen Herzfehlern (ausgenommen: thorakale Organtransplantation) kontinuierlich mit der gebotenen Routine zur Verfügung stellen. Darüber hinaus müssen diese herzchirurgischen Einheiten regelmäßige wissenschaftliche Aktivitäten nachweisen können und gewährleisten, dass in diesen Einheiten Weiter- und Fortbildung im Zusammenhang mit dem Facharzt für Herzchirurgie stattfindet. Die Verantwortlichen aller kinderherzchirurgischen Einheiten verpflichten sich zur Teilnahme an der gegenwärtig freiwilligen Nationalen Qualitätssicherung Angeborene Herzfehler von DGTHG und DGPK und führen zudem eine einrichtungsbezogene herzchirurgische Ergebnisbewertung mit Risikostratifizierung durch. Ergänzt wird dies durch die Bereitschaft individueller personenbezogener externer Zertifizierungen sowie die Ermöglichung von Peer Review Verfahren im Sinne der Qualitätssicherung. Darüber hinausgehende Maßnahmen wie die Kooperation der Einrichtungen zur klinischen Forschung, sowie die kontinuierliche fachgebiets- und berufsgruppenübergreifende Weiter- und Fortbildung sind wünschenswert. (c) 2016 Georg Thieme Verlag KG Stuttgart · New York
DOI http://dx.doi.org/ $10.1055 / \mathrm{s}-0035-1570099$. ISSN 0171-6425. 


\begin{abstract}
This document defines fundamental structures of congenital cardiac surgery departments in Germany. It has been developed by the executive boards of the German Society for Thoracic and Cardiovascular Surgery (GSTCVS) and the German Society of Pediatric Cardiology (GSPC) in collaboration with the working group for Congenital and Pediatric Heart Surgery of the GSTCVS.

This updated consensus paper is based on a previous publication of the European Association for Cardiothoracic Surgery (EACTS) and is a refinement and adaptation of its initial version published by the GSTCVS in 2005. In Germany, pediatric cardiology and cardiac surgery facilities caring for patients with congenital cardiac defects are subject to certain regulations. For example, in 2010 the Federal joint Committee implemented the resolution on Quality Assurance Measures in the Provision of Cardiac Surgical Care for Children and Adolescents (directive congenital cardiac surgery) which regulates structural and process quality compulsorily. To date, fundamental and considerable differences of the respective departments persist.

Congenital cardiac surgery departments have to provide the whole spectrum of the cardiac surgical therapy from the neonate to the adult with congenital cardiac defects (with the exception of heart transplantation) continuously and with the appropriate experience. Furthermore, the departments have to prove their constant scientific activity and ensure that they facilitate education and training for the specialty certification in cardiac surgery. The responsible surgeons of all congenital cardiac surgery departments commit to participate in the currently voluntary national quality assurance for congenital cardiac defects of the GSTCVS and the GSPC and perform an individual surgical outcome assessment and risk stratification. This is supplemented by the willingness for external certification specific to the individual and the facilitation of peer review procedures for quality assurance purposes. Additional measures, such as collaboration in clinical research and ongoing interdisciplinary education and training, are preferable.
\end{abstract}

\section{Einleitung}

Herzchirurgische Eingriffe bei Patienten mit angeborenen Herzfehlern bedürfen einer spezialisierten Expertise im Fachgebiet Herzchirurgie. Dies gilt, aufgrund der besonderen physiologischen Charakteristika von Früh-, Neugeborenen, Kindern und Jugendlichen, auch für die herzchirurgische Versorgung dieser Patienten mit erworbenen Herzfehlern. Der medizinische Fortschritt der letzten Jahrzehnte hat dazu geführt, dass diese Patienten frühzeitiger operativ oder interventionell behandelt werden können.

Wesentlich dazu beigetragen haben ein besseres Verständnis der (Patho-)Physiologie, eine genauere und damit aussagekräftigere Bildgebungsdiagnostik, Weiterentwicklungen und Innovationen der Herzkatheterverfahren, differenziertere intensivmedizinische Behandlungsoptionen und zahlreiche weiterentwickelte herzchirurgische Methoden. Durch all diese Faktoren konnten im Hinblick auf das Outcome der Patienten erhebliche Verbesserungen der Kurz- und insbesondere auch der Langzeitergebnisse erzielt werden. ${ }^{1}$ Begleitende Aktivitäten der wissenschaftlich-medizinischen Fachgesellschaften wie die personenbezogene Zertifizierung (DGTHG), die Einführung der nationalen Qualitätssicherung zur Behandlung angeborener Herzfehler (DGTHG u. DGPK) und die Zertifizierung von Zentren zur Behandlung von
Erwachsenen mit angeborenem Herzfehler [EMAH] (DGTHG, DGPK, DGK) haben zudem ihren Beitrag zur qualitativen Verbesserung der Patientenversorgung geleistet.

Die Chirurgie angeborener Herzfehler war ein Pionierbereich in der frühen Entwicklung der Herzchirurgie und ist daher seit jeher und bis heute ein wichtiger Teilbereich des herzchirurgischen Fachgebietes.

Angesichts der dargelegten Errungenschaften und der sich kontinuierlich verändernden Bedingungen in allen Bereichen des deutschen Gesundheitsheitswesens erscheint eine Aktualisierung des bisher geltenden Strukturpapiers notwendig bzw. geboten. Hierdurch soll sichergestellt werden, dass alle bei der Erstveröffentlichung im Jahr 2005 getroffenen Aussagen einer differenzierten Überprüfung unterzogen werden, um festzustellen ob sie unverändert gelten, angepasst bzw. ergänzt oder gänzlich revidiert werden müssen.

Dieses Konsensuspapier beruht somit in Grundzügen auf der Veröffentlichung „Optimal structure of a congenital heart surgery department in Europe“der European Association for Cardiothoracic Surgery (EACTS) aus dem Jahr $2003^{2}$ und dem im Jahr 2005 erstmals publizierten DGTHG-Strukturpapier „Struktur chirurgischer Einheiten zur Behandlung angeborener Herzfehler“3. 


\section{Ärztliche Kompetenz}

Der Begriff ärztliche Kompetenz subsummiert umfassende personenbezogene Eigenschaften, die von mentalen und kommunikativen Fähigkeiten über medizinische Kenntnisse bis hin zu praktischen Fertigkeiten reichten. Diese vielfältige Kompetenz gilt auch für den Bereich der Chirurgie angeborener Herzfehler, bzw. bei der herzchirurgischen Behandlung angeborener Fehlbildungen des Herzens, der Lungen und der großen herznahen Gefäße im Säuglings-, Kindes- und Jugendalter. Auch erworbene Herzerkrankungen in den zuvor genannten Alterskategorien wie z.B. Infektionen, Tumore oder kardiale Traumata gehören in diesen Bereich. Die notwendige herzchirurgische Expertise setzt weitreichende Kenntnisse in Diagnostik, differenzierter Indikationsstellung, Physiologie und Pathophysiologie des Herz-Kreislaufsystems und zu patientenindividuellen Therapieoptionen voraus. Ferner müssen in diesem Bereich tätige Herzchirurgen Kenntnisse zur Nachsorge bzw. Rehabilitation, häuslichen Versorgung sowie psychosozialen und ethischen Aspekten haben. Wesentliche Aspekte dieser umfassenden Kompetenz werden bei der personenbezogenen Zertifizierung der DGTHG „Chirurgie angeborener Herzfehler" berücksichtigt.

Im Detail sind folgende Voraussetzungen / Nachweise zur Antragstellung für das Zertifikat „Chirurgie angeborener Herzfehler" obligat:

i. Facharztqualifikation, Weiter- und Fortbildungszeiten

- Facharzt für Herzchirurgie

- Eine Fortbildungszeit im Bereich der Chirurgie angeborener Herzfehler von mindestens 36 Monaten, wobei 12 Monate aus der herzchirurgischen Facharztweiterbildung oder 12 Monate Fortbildung aus dem Fachgebiet pädiatrische Kardiologie anerkannt werden können.

ii. Praktische herzchirurgische Qualifikation inkl. Richtzahlen zu operativen Eingriffen bei

- Patienten mit einem Lebensalter $\leq 1$ Jahr 20 Herzoperationen unter Einsatz der Herz-Lungen-Maschine, 20 Herzoperationen ohne Einsatz der Herz-LungenMaschine

- Patienten mit einem Lebensalter $>1$ Jahr 40 Herzoperationen unter Einsatz der Herz-Lungen-Maschine

- Adoleszente Patienten ( $\geq 18$ Jahre) 20 Herzoperationen mit oder ohne Einsatz der Herz-Lungen-Maschine

iii. Eingehende Kenntnisse, Erfahrungen und Fertigkeiten

(Theorie und Praxis) in

- der Angio- und Sonographie des Herzens und der thorakalen Organe bei angeborenen Herzfehlern

- der operativen Behandlung von angeborenen Fehlbildungen und erworbenen Erkrankungen des Herzens im Säuglings-, Kindes- und Jugendalter

- der kinderherzchirurgischen/kinderkardiologischen Intensivmedizin

- der operativen und interventionellen Behandlung herznaher großer Gefäße mit/ohne extrakorporale Zirkulation

\section{- transvenösen und epikardialen Herzschrittmacher- - Defibrillator-Implantationen (AICD)}

Die Gültigkeit des Zertifikats ist begrenzt auf fünf Jahre ab dem Zeitpunkt des mündlichen Prüfungsgesprächs. Nach der Erst-Zertifizierung sind Rezertifizierungen in einem angepassten Antragsverfahren vorgesehen.

Zur kompetenten Entscheidungsfindung der herzchirurgischen Tätigkeit gehört u.a. auch eine kontinuierliche, interdisziplinäre Zusammenarbeit im qualifizierten Team für angeborene Herzfehler (pädiatrischer Kardiologe, zertifizierter Herzchirurg „Chirurgie angeborener Herzfehler“, zertifizierter Kardiologe „Erwachsene mit angeborenen Herzfehlern“). Die patientenindividuelle Behandlungsstrategie (herzchirurgisches oder katheterinterventionelles Verfahren, Hybridprozedur, konservative Therapie etc.) muss daher für jeden einzelnen Patienten interdisziplinär diskutiert sowie stets im Einvernehmen festgelegt werden. Auf dieser Grundlage kann dann mit dem Patienten oder dessen Sorgeberechtigten ein sog. ,informed consent“ erreicht werden. Selbstverständlich muss bei Eingriffen, die andere Fachgebiete betreffen (z.B Traumata, Tumorleiden) eine Zusammenarbeit mit den Fachärzten der entsprechenden Fachgebiete erfolgen.

\section{Infrastruktur}

Einheiten zur herzchirurgischen Behandlung angeborener Herzfehler sind in Deutschland heterogen organisiert. Das Spektrum reicht von mehr oder minder in herzchirurgische Fachabteilungen integrierte Bereiche / Sektionen bis hin zu eigenständigen, spezialisierten Abteilungen / Departments in einer Einrichtung. In Kooperation mit Fachabteilungen für pädiatrische Kardiologie sowie weiteren eingebundenen Fachabteilungen existieren zudem fachdisziplinübergreifende „Kinderherzzentren“.

Für die herzchirurgische Behandlung von Patienten mit angeborenen Herzfehlern muss mindestens ein dem technischen Fortschritt entsprechender Operationssaal mit für Kinder und Jugendliche geeigneter Herz-Lungen-Maschine, extrakorporaler Membranoxygenation, intraoperativer Echokardiographie, Röntgen- und Durchleuchtungsgeräten jederzeit verfügbar sein. Darüberhinaus sollte ein zusätzlicher Operationssaal für Notfälle verfügbar sein.

Eine fachgebundene pädiatrisch-kardiologische Intensiveinheit muss am Standort kontinuierlich verfügbar sein. Operationssaal und Intensiveinheit müssen in räumlicher Nähe liegen. Befindet sich die pädiatrisch-kardiologische Intensiveinheit integriert in eine interdisziplinäre Intensiveinheit, muss die Bündelung der Ressourcen in einem separaten fachspezifischen Bereich erfolgen.

Eine pädiatrisch-kardiologische Pflegestation ist ebenfalls ein unabdingbarer Bestandteil in der angemessenen Versorgung von Patienten mit angeborenen Herzfehlern.

Weiterhin ist ein für die Behandlung von Patienten mit angeborenen Herzfehlern ausgerüstetes pädiatrisch-kardiologisches Herzkatheterlabor notwendig. Dieses muss in räumlicher Nähe zur Intensiveinheit liegen. 
Komplexe bildgebende Diagnostik muss zur Verfügung stehen und es müssen Konsiliardienste aus folgenden Fachgebieten verfügbar sein: Pädiatrie, Kinderchirurgie, Kinderurologie, Neurochirurgie, Nephrologie, Hals-NasenOhrenheilkunde.

\section{Personal und Prozesse}

Die Personalstruktur in Einrichtungen, die herzchirurgische Eingriffe bei Patienten mit angeborenen Herzfehlern durchführen, muss eine kontinuierliche, qualitativ angemessene herzchirurgische Versorgung gemäß der Richtlinie für Kinderherzchirurgie $^{4}$ gewährleisten können.

Jede ärztliche Leitung einer Einheit zur herzchirurgischen Behandlung angeborener Herzfehler muss über ein gültiges Zertifikat „Chirurgie angeborener Herzfehler" der DGTHG und über mindestens 5 Jahre ärztliche Kompetenz für den Bereich angeborener Herzfehler verfügen. Zudem ist die ärztliche Leitung für die herzchirurgische Weiter- bzw. Fortbildung verantwortlich und hat nachweisbar wissenschaftlich gearbeitet. Ferner verantwortet die ärztliche Leitung die Prozesse der Operationsorganisation.

Personell muss die Patientenversorgung einer Einheit zur herzchirurgischen Behandlung angeborener Herzfehler durch mindestens zwei für die Behandlung angeborener Herzfehler qualifizierte und zertifizierte Fachärzte für Herzchirurgie gewährleistet sein. Diese beiden Fachärzte für Herzchirurgie tragen die Verantwortung für die Durchführung und die Organisation der herzchirurgischen Behandlung und sind kontinuierlich in die perioperative Versorgung der Patienten mit angeborenen Herzfehlern eingebunden.

Das herzchirurgische Operationsteam besteht mindestens aus einem Facharzt für Herzchirurgie mit Zertifikat „Chirurgie angeborener Herzfehler" und einem in der Facharztweiterbildung befindlichen Assistenzarzt, der jedoch bereits über Erfahrungen in der Chirurgie angeborener Herzfehler verfügen muss. Ferner gehören dem Operationsteam ein Facharzt für Anästhesiologie, ein zertifizierter Kardiotechniker (European Board for Cardiovascular Perfusion) und qualifiziertes OP-/Anästhesie-Pflegepersonal an, die allesamt im Kontext der herzchirurgischen Behandlung angeborener Herzfehler erfahren sind.

Darüber hinausgehende personelle Anforderungen (z.B. Pflegepersonal auf der Intensivstation) für Einrichtungen zur herzchirurgischen Versorgung von Kindern und Jugendlichen sind in der G-BA-Richtlinie „Kinderherzchirurgie“ in der Fassung vom 18. Februar 2010 einschließlich aktueller Änderungen vom 3. Dezember $2014^{4}$ festgelegt.

\section{Herzchirurgische Expertise}

Eine fundierte und evidenzbasierte Definition einer minimalen oder gar optimalen herzchirurgischen „operativen Aktivität" für entsprechende Einrichtungen oder bezogen auf die dort tätigen Herzchirurgen erscheint mitunter aufgrund der Sub-Spezialisierung des Fachgebietes Herzchirurgie kaum möglich. ${ }^{5-7}$ Daher ist es dringend erforderlich, eine einheitliche, differenzierte und verlässliche Qualitätssicher- ung als Beurteilungsgrundlage zu etabieren, um so eine institutionsbezogene Vergleichbarkeit zu ermöglichen und darauf aufbauend die bundesweiten Versorgungsstrukturen für Patienten mit angeborenen Herzfehlern weiter zu verbessern. Trotz der bisher eingeschränkten Zahlen, Daten und Fakten für diesen Versorgungsbereich muss sichergestellt werden, dass das Erwerben kinderherzchirurgischer Expertise und die Versorgung von Patienten mit angeborenen Herzfehlern in Deutschland nur mit Erfüllung der Anforderungen dieses Konsensuspapiers und der entsprechenden GBA Richtlinie ${ }^{4}$ erfolgen dürfen.

Ziel ist es, durch Bündelung der Expertise die Qualität der herzchirurgischen Versorgung angeborener Herzfehler in Deutschland nachhaltig weiter zu verbessern. Dabei gilt es festzustellen, daß diverse Publikationen „Mengen-QualitätsRelationen" dargelegt haben. Für den Bereich herzchirurgischer Eingriffe angeborener Herzfehler ist jedoch nur eine geringe Evidenz vorhanden, weshalb bisher sog. „Schwellenwerte“ auf wissenschaftlicher Grundlage nicht definiert werden konnten. Zudem geht eine größere Anzahl bzw. Menge von Leistungen nicht automatisch mit der $\mathrm{Zu}$ nahme von Qualität einher. Um eine angemessene Bewertung diverser Qualitätsaspekte zu ermöglichen, erscheint eine verpflichtende bundesweite Qualitätssicherung (s. 7. Qualitätssicherung) als ein geeignetes Instrument.

\section{Erwachsene mit angeborenen Herzfehlern (EMAH)}

Die Behandlungserfolge bei Patienten mit angeborenen Herzfehlern im Neugeborenen- und Kindesalter hat in den vergangenen Jahren zu einer zunehmenden Anzahl von erwachsenen Patienten geführt, die wegen residualer angeborener und/oder erworbener Herz- und /oder Gefäßerkrankungen behandelt werden müssen. ${ }^{8}$ Diese Patienten erfordern eine spezielle interdisziplinäre Betreuung durch Fachärzte für pädiatrische Kardiologie, Fachärzte für Kardiologie und Fachärzte für Herzchirurgie mit dem Zertifikat Chirurgie angeborene Herzfehler. Einige spezielle Anforderungen zu Infrastrukturen, Prozessen und fachärztlichen Qualifikationen wurden bereits in Empfehlungen und Positionspapieren publiziert und werden daher in diesem Konsensuspapier nicht erneut thematisiert. ${ }^{8,9}$

\section{Qualitätssicherung}

Zur Sicherung der Prozess- und Ergebnisqualität legt die G-BA Richtlinie zur Kinderherzchirurgie zahlreiche konkrete Anforderungen fest. ${ }^{4}$ Diese beinhalten bspw. vierteljährliche Teamsitzungen, einen regelmäßig tagenden abteilungs-übergreifenden, interdisziplinären, multiprofessionell zusammengesetzten Qualitätszirkel, die Bereitstellung schriftlicher Informationen an Sorgeberechtigte sowie die Ermöglichung des fachlichen Austausches über die Einrichtung hinaus.

Die Teilnahme an einer externen QualitätssicherungsMassnahme muss für jede Einheit zur Behandlung angeborener Herzfehler verpflichtend sein. Hierbei ist es ebenso obligat, daß alle in diesem Zusammenhang zu erhebenden 
Daten vollständig und valide erhoben sowie zur Verfügung gestellt werden müssen. Die Einführung einer gesetzlich verpflichtenden Qualitätssicherungs-Massnahme, bspw. nach § 137 SGB V, muss dringend erfolgen. Dazu ist ein differenziertes QS-Verfahren mit der Entwicklung geeigneter QS-Indikatoren notwendig. Die Analyse der Resultate muss regelmäßig, am besten durch eine unabhängige Institution sowie unter Einbeziehung fachlicher Expertise, durchgeführt werden. Hierbei sollten die Fachgesellschaften (DGTHG, DGPK), die bereits eine erste Initiative für eine nationale Qualitätssicherung angeborener Herzfehler gestartet haben, einbezogen werden. Um die Ergebnisse (z. B. im zeitlich longitudinalen Verlauf) einrichtungsbezogen differenziert bewerten und bundesweit vergleichen zu können, ist eine Risikostratifizierung zwingend erforderlich. ${ }^{10}$

Im Zusammenhang mit der invasiven Therapie angeborener Herzfehler wurden bereits mehrere Risiko-Score-Modelle auf internationaler Ebene erarbeitet und publiziert. So stehen in diesem Zusammenhang der Aristotle-, der RACHS-, der STSMobidity-Score (Jacobs-et al) oder auch das C3PO-Instrument (Bergersen-et al) zur Verfügung. ${ }^{10-14}$ Ergänzend zu den heutigen Qualitätssicherungsmaßnahmen sollten regelmäßige Analysen der behandlungsassoziierten Morbidität und eine Evaluation des Langzeit-Outcome im Sinne eines fachgebietsübergreifenden bzw. interdisziplinären Peer-Reviews erfolgen. Ziel sollte es zudem sein, durch eine strukturierte Kooperation der Einrichtungen die Weiterentwicklung von Behandlungsmethoden zu ermöglichen und deren Implementierung zu beschleunigen.

\section{Weiter- und Fortbildung}

Eine Einheit zur herzchirurgischen Behandlung angeborener Herzfehler sollte, abgesehen von der Infrastruktur für die Patientenversorgung, zusätzlich über adäquate Räumlichkeiten (z.B. Sitzungsräume), einen angemessenen Zugang zu medizinischen Bibliotheken, Datenbanken und webbasierten Weiterbildungsmöglichkeiten verfügen. Regelmäßige Weiter- und Fortbildungsveranstaltungen (Physiologie, Pathophysiologie, Pathologie, Herzchirurgie und Kinderkardiologie) sollten organisiert werden. Im Sinne der continuous medical education (CME) sind regelmäßige Postgraduiertenfortbildungen und Teilnahmen an nationalen und internationalen Kongressen für alle ärztlichen Teammitglieder obligat. Ferner sollte im Kontext der Facharztweiterbildung ein strukturiertes Weiterbildungsprogramm speziell für den Teilbereich angeborener Herzfehler vorhanden sein und die Weiterbildung möglichst das gesamte Spektrum der angeborenen Herzfehler umfassen. $^{15}$

Operative Eingriffe bei angeborenen Herzfehlern müssen bei der Weiterbildung zum „Facharzt für Herzchirurgie“ anrechenbar sein.

Nach Erreichen der Facharztqualifikation für Herzchirurgie existiert eine zusätzliche Qualifikationsmöglichkeit, die anhand eines speziellen Curriculums mit eigens festgelegter Weiter- bzw. Fortbildungszeit und einer mündlichen Prüfung den Erwerb des Zertifikats „Chirurgie für angeborene Herzfehler“ der DGTHG ermöglicht.

\section{Forschung}

Wissenschaftliche Forschung ist in einer herzchirurgischen Einheit zur Behandlung angeborener Herzfehler unabdingbar. In nicht universitären Einrichtungen sollte die Bereitschaft zur Teilnahme an klinischen Multizenterstudien selbstverständlich und eine Kooperation mit externen Forschungseinrichtungen möglich sein. Regelmäßige Veröffentlichungen in entsprechenden Fachzeitschriften sind wünschenswert. Auch Grundlagenforschung in Bereichen wie der Physiologie, Immunologie, Zellbiologie, Genetik, usw. kann in enger Zusammenarbeit mit anderen Forschungslabors erfolgen.

\section{Literaturverzeichnis}

1 Meinertz T, Diegeler A, Stiller B, et al. German Heart Report 2013. Thorac Cardiovasc Surg 2015;63(2):86-96

2 Daenen W, Lacour-Gayet F, Aberg T, et al; EACTS Congenital Heart Disease Committee. Optimal structure of a congenital heart surgery department in Europe. Eur J Cardiothorac Surg 2003;24(3): 343-351

3 Vorstand und AG Angeborene Herzfehler/Kinderherzchirurgie der DGTHG. Struktur chirurgischer Einheiten zur Behandlung angeborener Herzfehler. Thorac Cardiovasc Surg 2006;54(2): 73-77

4 Richtlinie zur Kinderherzchirurgie G-BA. Maßnahmen zur Qualitätssicherung der herzchirur-gischen Versorgung bei Kindern und Jugendlichen gemäß $§ 137$ Absatz 1 Nummer 2 SGB V Available at: https://www.g-ba.de/informationen/richtlinien/71

5 Jensen HA, Brown KL, Pagel C, Barron DJ, Franklin RC. Mortality as a measure of quality of care in infants with congenital cardiovascular malformations following surgery. Br Med Bull 2014;111(1): 5-15

6 Vinocur JM, Menk JS, Connett J, Moller JH, Kochilas LK. Surgical volume and center effects on early mortality after pediatric cardiac surgery: 25-year North American experience from a multi-institutional registry. Pediatr Cardiol 2013;34(5): 1226-1236

7 Welke KF, Karamlou T, Ungerleider RM, Diggs BS. Mortality rate is not a valid indicator of quality differences between pediatric cardiac surgical programs. Ann Thorac Surg 2010;89(1): 139-144, discussion 145-146

8 Hess J, Bauer U, de Haan F, et al. Empfehlungen für Erwachsenenund Kinderkardiologen zum Erwerb der Zusatz-Qualifikation "Erwachsene mit angeborenen Herzfehlern" (EMAH). Clin Res Cardiol Suppl 2007;2:19-26 DOI 10.1007/s11789-0060026-9

9 Kaemmerer H, Bauer U, de Haan F, et al. Recommendations for improving the quality of the interdisciplinary medical care of grown-ups with congenital heart disease (GUCH). Int J Cardiol 2011;150(1):59-64

10 Lacour-Gayet F, Clarke D, Jacobs J, et al; Aristotle Committee. The Aristotle score: a complexity-adjusted method to evaluate surgical results. Eur J Cardiothorac Surg 2004;25(6):911-924

11 Jenkins KJ. Risk adjustment for congenital heart surgery: the RACHS-1 method. Semin Thorac Cardiovasc Surg Pediatr Card Surg Annu 2004;7:180-184

12 O'Brien SM, Clarke DR, Jacobs JP, et al. An empirically based tool for analyzing mortality associated with congenital heart surgery. J Thorac Cardiovasc Surg 2009;138(5):1139-1153

13 Jacobs ML, O'Brien SM, Jacobs JP, et al. An empirically based tool for analyzing morbidity associated with operations for congenital heart disease. Ann Thorac Surg 2012;94:564-572 
24 Konsensuspapier Chirurgie Angeborener Herzfehler Beckmann

14 Bergersen L, Gauvreau K, Marshall A, et al. Cardiac Catheterization Procedure-Type Risk Categories for Pediatric and Congenital Cardiac Catheterization. Circ Cardiovasc Interv 2011;4:188-194
15 Lange R, Hoerer J, Schreiber C. What are the obstacles to training in surgery for congenital heart disease in Germany? Thorac Cardiovasc Surg 2013;61(4):273-277 